\title{
Parafuso pedicular: método para correção da deformidade na escoliose idiopática do adolescente
}

\author{
Pedicular screw: method to correct the deformity in adolescent \\ idiopatic scoliosis \\ Tornillo pedicular: método para corregir la deformidad en la \\ escoliosis idiopática del adolescente
}

\author{
Raphael de Rezende Pratali' \\ Marcelo Italo Risso Neto ${ }^{2}$ \\ Ivan Guidolin Veiga ${ }^{2}$ \\ Wagner Pasqualini² \\ Paulo Tadeu Maia Cavali ${ }^{3}$ \\ Marcus Alexandre Mello Santos 4 \\ Alexander Junqueira Rossato ${ }^{4}$ \\ Maurício Antonelli Lehoczki ${ }^{4}$ \\ Élcio Landim 5
}

\section{RESUMO}

Objetivo: avaliar retrospectivamente o poder de correção da deformidade escoliótica em pacientes tratados cirurgicamente com instrumental de terceira geração sendo utilizados exclusivamente parafusos pediculares tanto nas curvas torácicas quanto nas lombares. Métodos: dezessete pacientes com escoliose idiopática do adolescente (EIA) foram submetidos a tratamento cirúrgico, com correção da deformidade e artrodese via posterior com parafusos pediculares em todas as vértebras. Foram analisados sexo, idade, linha de Risser e classificação da escoliose pelo sistema de Lenke. As curvas foram comparadas entre si no pré e pós-operatório em relação ao grau da curvatura pelo método de

\section{ABSTRACT}

Objective: to evaluate retrospectively the power of scoliotic deformity in patients that were submitted to surgical treatment with third generation instrumentation using exclusively pedicle screws in both thoracic and lumbar curves. Methods: seventeen patients with adolescent idiopathic scoliosis (AIS) were submitted to surgical treatment with deformity correction and fusion by posterior approach with pedicle screws in all vertebral bodies. Sex, age, Risser line and Lenke system of classification were analyzed. The curves were compared between pre and postoperatory in relation to Cobb angle, curve flexibility by the bending radiographs, apical
\end{abstract}

\section{RESUMEN}

Objetivo: evaluar retrospectivamente la facultad de corregir la deformidad de escoliosis en los pacientes tratados quirúrgicamente con instrumento de tercera generación se utilizando exclusivamente los tornillos de pedículo, tanto en las curvas torácicas como en las lumbares. Métodos: diecisiete pacientes con escoliosis idiopática del adolescente fueron tratados quirúrgicamente, con la corrección de la deformidad y artrodesis posterior con tornillos pediculares en todas las vértebras. Se analizaron sexo, edad, línea de Risser y clasificación de la escoliosis por el sistema de Lenke. Las curvas fueron comparadas antes y después de la cirugía en relación con el grado de curvatura por el

\footnotetext{
Trabalho realizado no Hospital das Clínicas da Universidade Estadual de Campinas - UNICAMP - Campinas (SP), Brasil; e no Grupo de Escoliose da Associação de Assistência à Criança Deficiente - AACD - São Paulo (SP), Brasil.

'Estagiário de Coluna do Departamento de Ortopedia e Traumatologia da Universidade Estadual de Campinas - UNICAMP - Campinas (SP), Brasil. ${ }^{2}$ Cirurgião de Coluna do Departamento de Ortopedia e Traumatologia da Universidade Estadual de Campinas - UNICAMP - Campinas (SP), Brasil. ${ }^{3}$ Cirurgião de Coluna do Grupo de Escoliose da Associação de Assistência à Criança Deficiente - AACD - São Paulo (SP), Brasil; e do Departamento de Ortopedia e Traumatologia da Universidade Estadual de Campinas - UNICAMP - Campinas (SP), Brasil.

${ }^{4}$ Cirurgião de Coluna do Grupo de Escoliose da Associação de Assistência à Criança Deficiente - AACD - São Paulo (SP), Brasil. ${ }^{5}$ Chefe do Grupo de Escoliose da Associação de Assistência à Criança Deficiente - São Paulo (SP), Brasil; e Chefe do Grupo de Cirurgia da Coluna Vertebral do Departamento de Ortopedia e Traumatologia da Faculdade de Ciências Médicas da Universidade Estadual de Campinas - UNICAMP - Campinas (SP), Brasil. Recebido: 10/12/2009 Aprovado: 11/9/2010
} 
Cobb, sua flexibilidade por meio de radiografias em inclinação, translação vertebral apical (AVT, do inglês apical vertebral translation) e rotação vertebral apical (AVR, do inglês apical vertebral rotation). A porcentagem de correção pós-operatória foi analisada por meio do "índice de correção de Cincinnati” (ICC). Resultados: houve correção significativa no ângulo de Cobb nas curvas maiores $(p<0,0001)$ e nas curvas menores $(p<0,0001)$. O ICC obtido para as curvas maiores $(2,89)$ e menores $(1,21)$ foi significativamente diferente do zero $(\mathrm{p}=0,0143$ e $p<0,0001$, respectivamente). Em relação ao AVT, houve correção significativa nas curvas maiores ( $\mathrm{p}=0,0007)$, porém não-significativa nas curvas menores $(p=0,1082)$. O AVR foi corrigido significativamente tanto nas curvas maiores $(p=0,0001)$ quanto nas menores $(p=0,0033)$. Conclusão: o tratamento cirúrgico da EIA por artrodese via posterior com instrumental de terceira geração apresentou elevado poder de correção das deformidades, além de ter se mostrado técnica segura. vertebral translation (AVT) and apical vertebral rotation (AVR). The postoperative correction percentage was expressed by the Cincinnati correction index (CCI). Results: the Cobb angle had significant correction in the major curves $(p<0.0001)$ and the minor curves $(p<0.0001)$. The CCI of the major curves (2.89) and minor curves (1.21) differ significantly from zero $(p=0.0143$ and $p<0.0001$, respectively). The $A V T$ had significant correction in the major curves ( $p=0007)$, but not significant in the minor curves $(p=1.082)$. The AVR was significantly corrected in both major $(p=0.0001)$ and minor $(p=0.0033)$ curves. Conclusion: the surgery treatment of AIS with posterior fusion using third generation instrumentation has proved to be powerful in correcting the deformities besides shown to be a safe technique. método de Cobb, su flexibilidad con las radiografías en la pendiente, la traslación vertebral apical (AVT, sigla del inglés apical vertebral translation) y la rotación vertebral apical (AVR, del inglés apical vertebral rotation). El porcentaje de corrección postoperatoria fue analizado con el "índice de corrección de Cincinnati" (CPI). Resultados: se observó una corrección significativa en el ángulo de Cobb en las curvas de mayor $(p<0,0001)$ y en las curvas de menor $(p<0,0001)$. El CPI logrado para las curvas de mayor $(2,89)$ y menor $(1,21)$ era significativamente diferente de cero $(p=0,0143$ y $p<0,0001$, respectivamente). En relación con la AVT, hubo corrección significativa de las curvas de mayor ( $p=0,0007)$, pero no significativa en las curvas de menor ( $p=0,1082)$. El AVR se corrigió significativamente tanto en las curvas de mayor $(p=0,0001)$ como en la curva de menor $(p=0,0033)$. Conclusión: el tratamiento quirúrgico de la EIA por artrodesis posterior con la tercera generación de alta potencia instrumental presentó grande poder para corregir las deformidades, y ha demostrado ser una técnica segura.

DESCRIPTORES: Escoliosis;

Curvatura de la columna vertebral; Fusión vertebral/ métodos; Instrumentos quirúrgicos; Tornillos óseos

\section{INTRODUÇÃO}

Escoliose idiopática do adolescente (EIA) é uma deformidade complexa e tridimensional que afeta a coluna e a caixa torácica $^{1,2}$. Os casos que apresentam curva de maior magnitude e potencial de progressão requerem tratamento cirúrgico, baseado principalmente em artrodese associada à correção da deformidade. Atualmente, as técnicas cirúrgicas quase sempre se associam com instrumentação aumentando, assim, a estabilidade e oferecendo maior potencial de correção $0^{3,4}$. O objetivo do procedimento cirúrgico não consiste apenas em corrigir a deformidade, sendo ainda mais importante melhorar a postura e obter equilíbrio do eixo sagital e coronal aceitáveis com ombros alinhados e paralelos à pelve ${ }^{4}$.

Diversos sistemas de classificação para escoliose foram descritos, buscando categorizar modelos de deformidade e orientar um plano para instrumentação. O sistema de classificação mais popular é o proposto por King-Moe ${ }^{5}$ e mais recentemente a Classificação de Lenke $^{6}$ tem sido amplamente utilizada. Lenke estabeleceu que todo paciente com escoliose possui três curvas (torácica proximal, torácica principal e toracolombar/lombar), sendo a de maior mensuração, chamada curva maior, obrigatoriamente rígida. As outras duas curvas, chamadas menores, podem ser flexíveis, caso as radiografias de inclinação lateral corrijam para valor inferior a $25^{\circ}$, ou rígidas, caso não corrijam para menos de $25^{\circ}$ e/ou apresentem uma cifose maior que $20^{\circ}$. No sistema de Lenke, além da deformidade no plano coronal, também se considera a deformidade torácica no plano sagital (modificador sagital) e a translação da vértebra apical da curva lombar (modificador lombar). O sistema de 
Lenke tem sido mais preciso para guiar níveis de fusão no tratamento da escoliose.

Os sistemas de instrumentação modernos multissegmentares envolvem numerosas opções para fixação espinhal e permitem técnicas diferentes entre si para correção da deformidade escoliótica ${ }^{4}$.

Desde a introdução da instrumentação de CotrellDubousset (CD) em 1984, as técnicas de correção da escoliose têm mudado dos princípios de Harrington, que envolve distração da concavidade, para realinhamento segmentar incluindo várias possibilidades como manobra de rotação da haste e aproximação segmentar pelo método de "cantilever"8-10.

Adicionalmente, utilização de parafusos pediculares nas curvas lombares melhorou a correção e estabilização de várias deformidades, sendo que vários trabalhos demonstraram vantagem clínica do parafuso pedicular lombar sobre instrumentação convencional com ganchos ${ }^{11,12}$. Mais recentemente, vários autores relataram o uso dos parafusos pediculares em curvas torácicas, obtendo melhor correção das deformidades e permitindo construções mais $\operatorname{curtas}^{13,14}$. A maioria desses autores relataram não haver aumento no risco de lesão neurológica com uso de parafusos pediculares torácicos ${ }^{13-16}$.

Instrumentação segmentar com parafusos pediculares permite melhor correção das deformidades nos planos coronal, sagital e rotacional, menor perda de redução, construções mais curtas e melhora na função pulmonar, sem aumento das complicações neurológicas, comparado com instrumentação com ganchos ou híbrida (ganchos proximais e parafusos pediculares distais) $)^{17,18}$.

O objetivo deste trabalho foi avaliar retrospectivamente o poder de correção da deformidade escoliótica em pacientes tratados cirurgicamente com instrumental de terceira geração, sendo utilizados exclusivamente parafusos pediculares tanto nas curvas torácicas quanto nas lombares.

\section{MÉTODOS}

Foram analisados 17 pacientes submetidos a tratamento cirúrgico para EIA, sendo os procedimentos realizados entre junho de 2005 e janeiro de 2009 no Hospital das Clínicas da Universidade Estadual de Campinas (UNICAMP) e na Associação de Assistência à Criança Deficiente (AACD). Do total de pacientes, 16 eram do sexo feminino e apenas um do sexo masculino. Suas idades variaram entre 11 e 21 anos (média de 14,6 anos).

No estudo foram considerados sexo, idade, linha de Risser e classificação da escoliose pelo sistema de Lenke. As curvas maiores e menores no mesmo paciente foram separadamente comparadas entre si, no pré e pós-operatório em relação ao grau da curvatura pelo método de Cobb, sua flexibilidade por meio de radiografias em inclinação, translação vertebral apical (AVT - apical vertebra translation) e rotação vertebral apical (AVR - apical vertebra rotation). Esses critérios complementam o sistema de classificação da escoliose de Lenke na decisão de realizar fusão seletiva ${ }^{19}$. A AVT das curvas torácicas principais é medida pela distância entre a linha de prumo de C7 e o ponto médio do corpo ou disco apical. Nas curvas toracolombares/lombares a AVT é obtida pela distância entre a linha vertical sacral média e o ponto médio do corpo ou disco apical. A AVR é medida no ápice de ambas as curvas pelo sistema de avaliação de Nash-Moe ${ }^{20}$.

Analisamos a porcentagem de correção pós-operatória, tanto nas curvas maiores quanto menores, por meio do "Índice de Correção de Cincinnati" (ICC) ${ }^{21}$, que leva em consideração todos os fatores significantes da curva escoliótica, como sua magnitude e principalmente sua flexibilidade. Trata-se de uma expressão matemática que corresponde à "correção pós-operatória" dividida pela "flexibilidade pré-operatória". A "Correção Pós-Operatória" é determinada pelo ângulo de Cobb pré-operatório menos o ângulo de Cobb pós-operatório dividido pelo ângulo de Cobb pré-operatório. A "flexibilidade pré-operatória" é determinada pelo ângulo de Cobb pré-operatório menos o ângulo de Cobb na inclinação dividido pelo ângulo de Cobb pré-operatório.

Todos os pacientes foram submetidos a tratamento cirúrgico da EIA realizado por cirurgiões da mesma equipe. Os procedimentos envolveram abordagem exclusivamente posterior para correção da deformidade, com liberação dos elementos posteriores e correção por meio de manobras de translação da vértebra instumentada, associado à distração da concavidade e compressão da convexidade quando necessárias, seguido por artrodese posterior. Foram utilizados exclusivamente parafusos pediculares tanto nas curvas lombares quanto nas curvas torácicas. Quanto ao pós-operatório, seguiu-se o mesmo protocolo para todos os pacientes, com início de marcha precocemente, sem uso de órteses.

As radiografias consideradas para a análise dos parâmetros pós-operatórios foram obtidas após o terceiro mês de cirurgia, sendo realizadas todas as medidas e comparadas com os parâmetros das radiografias pré-operatória. As medidas de todos os parâmetros foram realizadas pelo mesmo observador (Figura 1). Os valores dos parâmetros pré e pós-operatórios foram então submetidos à análise estatística. A análise dos parâmetros comparados entre si foi realizada através do teste $t$ de student.

\section{RESULTADOS}

Dos 17 pacientes analisados, $16(94,1 \%)$ eram do sexo feminino e um $(5,9 \%)$ do sexo masculino. Suas idades variaram entre 11 e 21 anos com média de 14,6 anos. A linha de Risser variou entre um caso (5,9\%) Risser zero; nenhum caso, Risser 1, dois casos $(11,7 \%)$ Risser 2, três casos $(17,6 \%)$ Risser 3, oito casos $(47 \%)$ Risser 4 e três casos $(17,6 \%)$ Risser 5. A média obtida referente à linha de Risser foi 3,5 (Tabela1).

Quanto à classificação de Lenke (Tabela 1), em relação ao padrão da curva no plano coronal, o número de pacientes foi: oito $(47 \%)$ do tipo 1 , nenhum tipo 2 , três $(17,6 \%)$ 

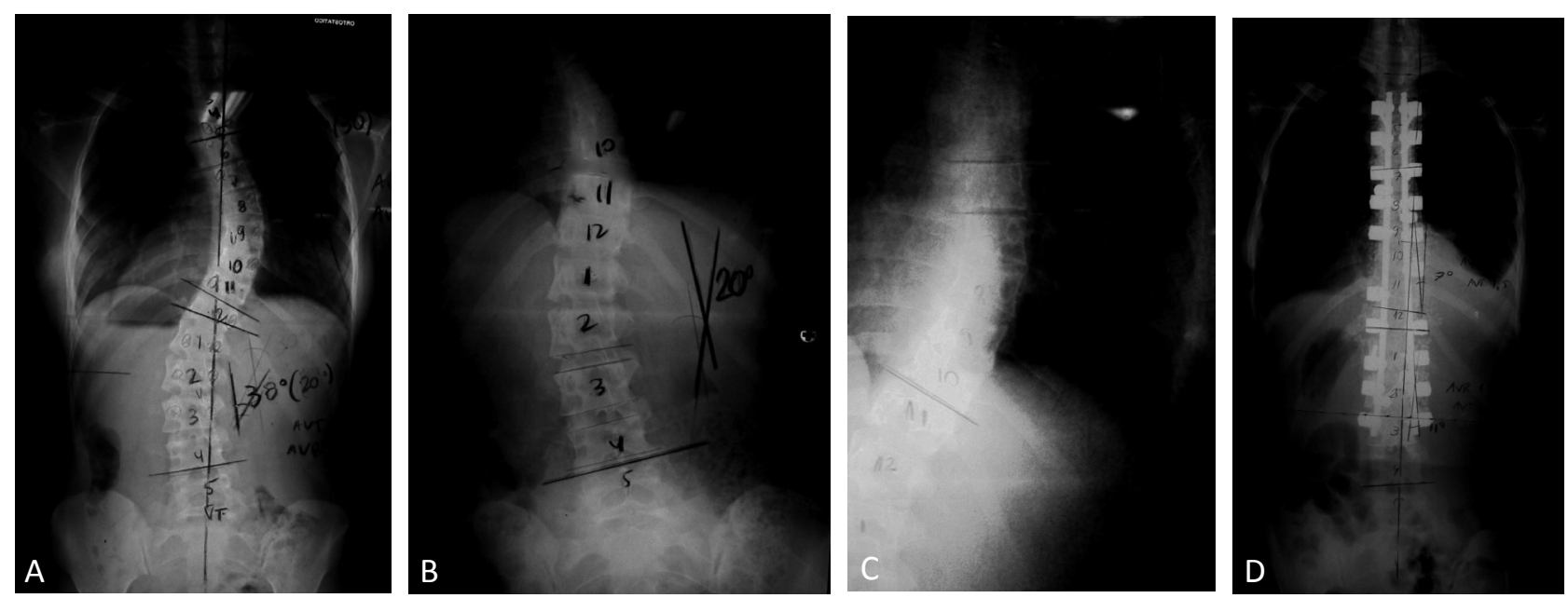

Figura 1

Análise dos parâmetros estudados nas radiografias pré e pós-operatórias: A) radiografia ortostática pré-operatória mostrando deformidade Lenke 1BN, com curva torácica principal (curva maior) T5-T12 direita de 44, AVT de 13 mm e AVR de 2 e curva lombar (curva menor) T12-L4 esquerda de 38, AVT de $11 \mathrm{~mm}$ e AVR de 2; B) radiografia em inclinação para direita mostrando correção da curva torácica principal para $30^{\circ}$; C) radiografia em inclinação para esquerda com correção da curva lombar para $20^{\circ}$; D) radiografia ortostática pós-operatória mostrando artrodese T5-L3, com correção da curva torácica para $7^{\circ}$, AVT de 15 $\mathrm{mm}$ e AVR de zero e da curva lombar para $11^{\circ}$, AVT de $5 \mathrm{~mm}$ e AVR de 1.

\section{TABELA 1 - Dados sobre os 17 pacientes com escoliose idiopática do adolescente (EIA) considerados para o estudo}

\begin{tabular}{lcccccc}
\hline Paciente & Sexo & Idade & Risser & Lenke & Número de vértebras & Número de parafusos \\
\hline 1 & $\mathrm{~F}$ & 12 & 2 & $6 \mathrm{CN}$ & 13 & 18 \\
2 & $\mathrm{~F}$ & 16 & 4 & $5 \mathrm{CN}$ & 10 & 17 \\
3 & $\mathrm{~F}$ & 13 & 3 & $1 \mathrm{BN}$ & 10 & 20 \\
4 & $\mathrm{~F}$ & 15 & 4 & $6 \mathrm{CN}$ & 6 & 12 \\
5 & $\mathrm{~F}$ & 18 & 5 & $3 \mathrm{BN}$ & 14 & 18 \\
6 & $\mathrm{~F}$ & 14 & 3 & $1 \mathrm{BN}$ & 10 & 13 \\
7 & $\mathrm{~F}$ & 14 & 4 & $4 \mathrm{C}+$ & 12 & 16 \\
8 & $\mathrm{~F}$ & 12 & 4 & $1 \mathrm{AN}$ & 8 & 13 \\
9 & $\mathrm{~F}$ & 18 & 4 & $1 \mathrm{BN}$ & 10 & 16 \\
10 & $\mathrm{~F}$ & 14 & 4 & $3 \mathrm{CN}$ & 13 & 13 \\
11 & $\mathrm{~F}$ & 14 & 4 & $5 \mathrm{CN}$ & 14 & 18 \\
12 & $\mathrm{~F}$ & 16 & 4 & $1 \mathrm{CN}$ & 13 & 13 \\
13 & $\mathrm{~F}$ & 21 & 5 & $1 \mathrm{BN}$ & 11 & 11 \\
14 & $\mathrm{~F}$ & 21 & 5 & $1 \mathrm{CN}$ & 7 & 11 \\
15 & $\mathrm{~F}$ & 11 & 0 & $5 \mathrm{CN}$ & 6 & 13 \\
16 & $\mathrm{M}$ & 14 & 2 & $6 \mathrm{C}+$ & 13 & 13 \\
17 & $\mathrm{~F}$ & 13 & 3 & $1 \mathrm{AN}$ & 10 & 13 \\
\hline
\end{tabular}

tipo 3 , um $(5,9 \%)$ tipo 4 , três $(17,6 \%)$ tipo 5 e dois $(11,7 \%)$ tipo 6 . Em relação ao modificador lombar, dois pacientes $(11,7 \%)$ eram tipo A, cinco $(29,4 \%)$ tipo B e dez $(58,9 \%)$ tipo C. Quanto ao modificador torácico que considera o alinhamento sagital, 16 pacientes $(94,1 \%)$ eram normocifóticos ("N"), um caso (5,9\%) tinha hipercifose ("+") e nenhum hipocifose (“-).

O número de vértebras envolvidas na artrodese variou entre 6 e 14, com média de 10,6 vértebras. A quantidade de parafusos pediculares utilizados para cada paciente, o que corresponde ao número de pedículos instrumentados, variou entre 11 e 22, com média de 15,9 parafusos (Tabela 1).
Os valores dos parâmetros medidos nas curvas maiores são mostrados na tabela 2 . O valor do ângulo de Cobb préoperatório variou entre $41^{\circ}$ e $77^{\circ}$ (média $53,4^{\circ}$ ), enquanto no pós-operatório entre $2^{\circ}$ e $50^{\circ}$ (média $15.2^{\circ}$ ), com $\mathrm{p}<0,0001$. O ICC variou entre 0,76 e 19,50 (média 2,89). O AVT préoperatório variou entre $11 \mathrm{~mm}$ e $62 \mathrm{~mm}$, com média de 31,4 $\mathrm{mm}$, enquanto no pós-operatório variou entre $3 \mathrm{~mm}$ e 56 $\mathrm{mm}$ com média de $13,1(\mathrm{p}<0,0001)$. O AVR pré-operatório variou entre 1 e 3 com média de 2, 1 , enquanto no pós-operatório variou entre 0 e 2 , média de $1,2(p=0,0001)$.

Para as curvas menores, os valores são mostrados na tabela 3. O ângulo de Cobb pré-operatório variou entre 
TABELA 2 - Parâmetros medidos referentes às curvas maiores

\begin{tabular}{lcccc}
\hline & Cobb & ICC & $\begin{array}{c}\text { AVT } \\
(\mathbf{m m})\end{array}$ & AVR \\
\hline Pré-operatório & $53.4^{\circ}$ & $-\cdots---$ & 31.4 & 2.1 \\
Pós-operatório & $15.2^{\circ}$ & 2.89 & 13.1 & 1.2 \\
Valor p & $<0.0001$ & 0.0143 & 0.0007 & 0.0001 \\
\hline
\end{tabular}

TABELA 3 - Parâmetros medidos referentes às curvas menores

\begin{tabular}{lcccc}
\hline & Cobb & ICC & $\begin{array}{c}\text { AVT } \\
(\mathrm{mm})\end{array}$ & AVR \\
\hline Pré-operatório & 37 & $-\cdots---$ & 15,1 & 1.4 \\
Pós-operatório & 11 & 1,21 & 9,2 & 0.7 \\
Valorp & $<0,0001$ & $<0,0001$ & 0,108 & 0,003 \\
\hline
\end{tabular}

$18^{\circ}$ e $62^{\circ}$ (média de $37^{\circ}$ ), enquanto no pós-operatório entre $0^{\circ}$ e $30^{\circ}$ (média $11^{\circ}$ ), com $\mathrm{p}<0,001$. O ICC variou entre 0,36 e 2,87 (média 1,21) O AVT pré-operatório variou entre 0 e $60 \mathrm{~mm}$, com média de $15,1 \mathrm{~mm}$, enquanto no pós-operatório entre 2 e $21 \mathrm{~mm}$, com média de 9,2 $\mathrm{mm}(\mathrm{p}=0,1082)$. O AVR pré-operatório variou entre $0 \mathrm{e}$ 3 com média de 1,4 e no pós-operatório entre zero e 2 , média de $0,7(\mathrm{p}=0,33)$.

Como complicações, um paciente apresentou soltura do parafuso pedicular do pedículo direito da vértebra mais distal (L2), apresentando dor no local, totalmente resolvido após retirada desse parafuso (demais instrumental não foi removido). Outro paciente mostrou sinais radiográficos sugestivos de soltura dos parafusos bilaterais da vértebra mais distal (L4), porém como se manteve assintomática nenhuma medida foi necessária. Não houve nenhum caso de infecção profunda, assim como nenhum caso de complicação neurológica.

\section{DISCUSSÃO}

Escoliose idiopática do adolescente é uma deformidade complexa cuja correção depende de inúmeros fatores. Os fatores que determinam a capacidade de correção cirúrgica por via posterior da EIA incluem a magnitude da curva, flexibilidade da curva, extensão da liberação cirúrgica dos elementos posteriores, número dos pontos de fixação e determinação da vértebra estável ${ }^{21}$. Nesse estudo, todos os pacientes foram submetidos à liberação cirúrgica de maneira similar. As técnicas de correção da deformidade também foram semelhantes, envolvendo translação da vértebra instrumentada e compressão da convexidade e distração da concavidade.

Com o advento dos sistemas de terceira geração ${ }^{22}$, a capacidade de o instrumental potencializar a correção da curva escoliótica aumentou drasticamente. Nos últimos anos, construções com parafusos pediculares ganharam popularidade ao ser percebido seu poder de correção superior. Quanto ao plano sagital, também se tem demonstrado que construções com parafusos pediculares fornecem melhor correção da cifose/lordose das curvas ${ }^{13,23}$.

Muitas outras vantagens têm sido relatadas em relação ao uso de construções com parafusos pediculares além do seu potencial de correção das deformidades. Isso inclui a possibilidade de poupar mais níveis de fusão comparada com outras técnicas, reduzir a necessidade de liberação de elementos vertebrais anteriores e da artodese por via anterior, proporcionar maior poder de fixação com menores taxas de revisão e melhorar a função pulmonar associada com melhor correção da deformidade torácica ${ }^{18,24}$.

Para avaliar o poder de correção da deformidade com o uso do material de terceira geração, utilizamos o Índice de Correção de Cincinnati, em vez da porcentagem de correção pós-operatória. Uma elevada porcentagem de correção obtida por uma construção pode, na verdade, estar associada a uma elevada flexibilidade da curva em relação a sua magnitude. Todos esses fatores são considerados na expressão matemática que leva à obtenção do ICC, tornando-o mais fidedigno para mensurar o poder de correção da curva escoliótica ${ }^{21}$.

Frente aos resultados obtidos nessa série, observamos que o tratamento cirúrgico utilizado mostrou elevado poder de correção, sendo obtida correção significativa no ângulo de Cobb tanto nas curvas maiores $(\mathrm{p}<0,0001)$ quanto nas curvas menores $(\mathrm{p}<0,0001)$. O ICC obtido para as curvas maiores $(2,89)$ e menores $(1,21)$ foi significativamente diferente do zero ( $\mathrm{p}=0,0143$ e $\mathrm{p}<0,0001$, respectivamente). Em relação ao AVT, houve correção significativa nas curvas maiores $(\mathrm{p}=0,0007)$, porém não-significativa nas curvas menores $(p=0,1082)$. O AVR foi corrigido significativamente tanto nas curvas maiores $(\mathrm{p}=0,0001)$ quanto nas menores $(\mathrm{p}=0,0033)$.

A taxa de complicações obtida foi muito reduzida, sendo que nenhuma dessas complicações prejudicou o resultado do tratamento ou afetou a satisfação pessoal dos pacientes, de modo que todos realizariam novamente o procedimento ou o indicariam a outros pacientes.

\section{CONCLUSÃO}

O tratamento cirúrgico da EIA, realizado por artodese via posterior com instrumental de terceira geração, apresenta elevado poder de correção das deformidades. Foi demonstrada correção significativa dos parâmetros analisados tanto em relação às curvas maiores quanto às curvas menores, com exceção do AVT nas curvas menores. Além disso, trata-se de uma técnica segura e com baixos índices de complicações. 


\section{REFERÊNCIAS}

1. Strokes IA, Bigalow LC, Moreland MS. Three-dimensional spinal curvature in idiopathic scoliosis. J Orthop Res. 1987;5(1):102-13.

2. Villemure I, Aubin CE, Grimard G, et al. Progression of vertebral and spinal three dimensional deformities in adolescent idiopathic scoliosis. Spine (Phila Pa 1976). 2001;26(20):2244-50.

3. Moe JH. Methods of correction and surgical techniques in scoliosis. Orthop Clin North Am. 1972;3(1):1748.

4. Bridwell KH. Surgical treatment of idiopathic adolescent scoliosis. Spine (Phila Pa 1976). 1999;24(24):2607-16.

5. King HA, Moe JH, Bradford DS, Winter RB. The selection of fusion levels in thoracic idiopathic scoliosis. J Bone Joint Surg Am. 1983;65(9):130213.

6. Lenke LG, Betz RR, Harms J, Bridwell KH, Clements DH, Lowe TG, et al. Adolescent idiopathic scoliosis: a new classification to determine extent of spinal arthrodesis. J Bone Joint Surg Am. 2001;83$\mathrm{A}(8): 1169-81$.

7. Vialle EN, Vialle LR, Martins Filho DE, Jorge RM. Confiabilidade de uma versão em português da classificação de Lenke para Escoliose Idiopática do Adolescente. Coluna/Columna. 2006;5(2):77-83.

8. Cotrel Y, Dubousset J. A new technique for segmental spinal osteosynthesis using the posterior approach. Rev Chir Orthop Reparatrice Appar Mot. 1984;70:48994.

9. Harrington PR. Treatment of scoliosis: correction and internal fixation by spine instrumentation. J Bone Joint Surg Am. 1962;44:591-610.

10. Cotrel Y, Dubousset J, Guillaumat M. New universal instrumentation in spinal surgery. Clin Orthop Relat Res. 1988;227:10-23.
11. Brown CA, Lenke LG, Bridwell KH, Geideman WM, Hasan SA, Blanke K. Complications of pediatric thoracolumbar and lumbar pedicle screws. Spine (Phila Pa 1976). 1998;23(14):1566-71.

12.Hamill CL, Lenke LG, Bridwell KH, Chapman MP, Blanke K, Baldus C. The use of pedicle screw fixation to improve correction in the lumbar spine of patients with idiopathic scoliosis. Spine. 1996;21(10):1241-9.

13.Suk SI, Lee CK, Kim WJ, Chung YJ, Park YB. Segmental pedicle screw fixation in the treatment of thoracic idiopathic scoliosis. Spine (Phila Pa 1976). 1995;20(12):1399-405.

14.Suk SI, Kim W, Lee S, Kim JH, Chung ER. Thoracic pedicle screw fixation in spinal deformities: are they really safe? Spine (Phila Pa 1976). 2001;26(18):2049-57.

15.Belmont PJ Jr, Klemme WR, Dhawan A, Polly DW Jr. In vivo accuracy of thoracic pedicle screws. Spine (Phila Pa 1976). 2001;26(21):2340-6.

16.Kim YJ, Lenke LG, Bridwell KH, Cho YS, Riew KD. Free hand pedicle screw placement in the thoracic spine: is it safe? Spine (Phila $\mathrm{Pa}$ 1976). 2004;29(3):333-42.

17.Kim YJ, Lenke LG, Cho SK, Briswell KH, Sides B, Blanke K. Comparative analysis of pedicle screw versus hook instrumentation in posterior spinal fusion of adolescent idiopathic scoliosis. Spine (Phila Pa 1976). 2004;29(18):2040-8.

18.Kim YJ, Lenke LG, Kim J, Bridwell $\mathrm{KH}$, Cho SK, Cheh G, et al. Comparative analysis of pedicle screw versus hybrid instrumentation in posterior spinal fusion of adolescent idiopathic scoliosis. Spine (Phila Pa 1976). 2006;31(3):291-8.
19.Lenke LG, Edwards CC, Bridwell KH. The Lenke classification of adolescent idiopathic scoliosis: how it organizes curve patterns as a template to perform selective fusions of the spine. Spine (Phila Pa 1976). 2003;28(20):S199-S207.

20.Nash CL Jr, Moe JH. A study of vertebral rotation. J Bone Joint Surg Am. 1969;51(2):223-9.

21. Vora V, Crawford A, Babekhir N, Boachie-Adjei O, Lenke L, Peskin M, et al. A pedicle screw construct gives an enhanced posterior correction of adolescent idiopathic scoliosis when compared with other constructs: myth or reality. Spine (Phila Pa). 2007;32(17):1869-74.

22.Lenke LG, Bridwell KH, Baldus C, Blanke K, Schoenecker PL. CotrelDubousset instrumentation for adolescent idiopathic scoliosis. J Bone Joint Surg Am. 1992;74(7):1056-67.

23.Lee SM, Suk SI, Chung ER. Direct vertebral rotation: a new technique of three-dimensional deformity correction with segmental pedicle screw fixation in adolescent idiopathic scoliosis. Spine (Phila Pa 1976). 2004;29(3):343-9.

24.Dobbs MB, Lenke LG, Kim YJ, Kamath G, Peelle MW, Bridwell Kh. Selective posterior thoracic fusions for adolescent idiopathic scoliosis: comparison of hooks versus pedicle screws. Spine (Phila Pa 1976). 2006;31(20):2400-4.

\section{Correspondência}

Raphael de Rezende Pratali

Rua Barata Ribeiro, 237 - Conjunto 66

CEP: 01308-000 - Bela Vista -

São Paulo (SP), Brasil

Telefone: (1 1) 2365-6638

E-mail:rpratali@fcm.unicamp.br 